\title{
Auxílio do intensificador de imagem na remoção de agulha gengival curta
}

\author{
Image intensifier aid in removing short gingival needlle \\ El intensificador de imágenes ayuda a retirar la aguija gingival corta
}

Recebido: 11/01/2021 | Revisado: 13/01/2021 | Aceito: 17/01/2021 | Publicado: 21/01/2021

\author{
Natália dos Santos Sanches \\ ORCID: https://orcid.org/0000-0003-07729-6505 \\ Universidade Estadual Paulista, Brasil \\ E-mail: naahssanches@gmail.com \\ Mirela Caroline Silva \\ ORCID: https://orcid.org/0000-0002-9455-3807 \\ Universidade Estadual Paulista, Brasil \\ E-mail: mirela_carol12@hotmail.com \\ William Phillip Pereira da Silva \\ ORCID: https://orcid.org/0000-0003-4172-7217 \\ Universidade Estadual Paulista, Brasil \\ E-mail: william_phillip@hotmail.com \\ Lara Cristina Cunha Cervantes \\ ORCID: https://orcid.org/0000-0003-4448-2702 \\ Universidade Estadual Paulista, Brasil \\ E-mail: laraccerv@gmail.com \\ Tiburtino José de Lima Neto \\ ORCID: https://orcid.org/0000-0002-8297-4057 \\ Universidade Estadual Paulista, Brasil \\ E-mail: tiburtinoneto@hotmail.com \\ Francisley Avila Souza \\ ORCID: https://orcid.org/0000-0002-1427-071X \\ Universidade Estadual Paulista, Brasil \\ E-mail: francisley.avila@unesp.br \\ Idelmo Rangel Garcia Júnior \\ ORCID: https://orcid.org/0000-0001-8892-781X \\ Universidade Estadual Paulista, Brasil \\ E-mail: irgcirurgia@gmail.com \\ Leonardo Perez Faverani \\ ORCID: https://orcid.org/0000-0003-2249-3048 \\ Universidade Estadual Paulista, Brasil \\ E-mail: leonardo.faverani@unesp.br
}

\begin{abstract}
Resumo
Fundamento: Mesmo que intercorrências envolvendo agulhas odontológicas atualmente sejam raras, acidentes são passiveis de ocorrer, principalmente em bloqueio do nervo alveolar inferior devido a inúmeros fatores inerentes ao operador e/ou o próprio material. O manejo desses pacientes visa a remoção da agulha imediatamente, se a ponta estiver visível, ou aplicação de métodos para localização da agulha no espaço pterigomandibular. Esses métodos vão desde radiografias simples à uso de scanners modernos, visando sempre o conforto do paciente e resolução rápida do quadro. Objetivo e relato de caso: O objetivo deste artigo é relatar um caso clínico da utilização do intensificador de imagem para remoção de agulha fraturada após anestesia do nervo alveolar inferior, durante um procedimento de exodontia. Paciente masculino, 28 anos, encaminhado ao serviço de emergência, sem queixas álgicas. A remoção cirúrgica com o auxílio do intensificador de imagem da agulha gengival fraturada alojada no ramo ascendente mandibular do lado esquerdo, foi realizada sob anestesia geral, por meio de um acesso em região de mucosa jugal. Conclusão: Para prevenção de fraturas de agulhas gengivais em procedimentos odontológicos, é necessário realizar a correta técnica anestésica, evitando dobras nas agulhas. Em caso de fraturas de agulhas, podemos concluir, que métodos adicionais, como a utilização do intensificador de imagem, auxilia a remoção ocasionando menores danos teciduais, consequentemente uma menor morbidade ao paciente.
\end{abstract}

Palavras-chave: Corpo estranho; Agulha; Traumatologia.

\begin{abstract}
Background: Even though complications involving dental needles are currently rare, accidents are likely to occur, mainly in lower alveolar nerve block due to numerous factors inherent to the operator and / or the material itself. The management of these patients aims to remove the needle immediately, if the tip is visible, or to apply methods to locate the needle in the pterygomandibular space. These methods range from simple radiographs to the use of modern
\end{abstract}


scanners, always aiming at patient comfort and quick resolution of the condition. Objective and case report: The objective of this article is to report a clinical case of the use of the image intensifier to remove a fractured needle after anesthesia of the lower alveolar nerve, during an extraction procedure. Male patient, 28 years old, referred to the emergency department, without pain complaints. Surgical removal with the aid of the image intensifier of the fractured gingival needle lodged in the left mandibular ascending branch, was performed under general anesthesia, by means of an access in the region of the jugal mucosa. Conclusion: To prevent fractures of gingival needles in dental procedures, it is necessary to perform the correct anesthetic technique, avoiding needle bends. In the case of needle fractures, we can conclude that additional methods, such as the use of the image intensifier, help the removal causing less tissue damage, consequently less patient morbidity.

Keywords: Strange body; Needle; Traumatology.

\section{Resumen}

Antecedentes: aunque en la actualidad las complicaciones relacionadas con las agujas dentales son poco frecuentes, es probable que ocurran accidentes, principalmente en el bloqueo del nervio alveolar inferior debido a numerosos factores inherentes al operador y / o al propio material. El manejo de estos pacientes tiene como objetivo retirar la aguja inmediatamente, si la punta es visible, o aplicar métodos para ubicar la aguja en el espacio pterigomandibular. Estos métodos van desde radiografías simples hasta el uso de escáneres modernos, siempre apuntando a la comodidad del paciente y la resolución rápida de la condición. Objetivo y reporte de caso: El objetivo de este artículo es reportar un caso clínico de uso del intensificador de imágenes para remover una aguja fracturada luego de la anestesia del nervio alveolar inferior, durante un procedimiento de extracción. Paciente varón de 28 años, remitido a urgencias, sin quejas de dolor. La extirpación quirúrgica con ayuda del intensificador de imágenes de la aguja gingival fracturada alojada en la rama ascendente mandibular izquierda, se realizó bajo anestesia general, mediante un acceso en la región de la mucosa yugal. Conclusión: Para prevenir fracturas de agujas gingivales en procedimientos dentales, es necesario realizar la técnica anestésica correcta, evitando que la aguja se doble. En el caso de las fracturas de agujas, podemos concluir que métodos adicionales, como el uso del intensificador de imágenes, ayudan a la extracción provocando menos daño tisular y, en consecuencia, menor morbilidad del paciente.

Palabras clave: Cuerpo extraño; Aguja; Traumatología.

\section{Introdução}

A partir do advento das agulhas descartáveis de ligas modernas, as fraturas de agulhas odontológicas em procedimentos de anestesia local em região maxilofacial tornaram-se não habituais (Ethunandan et al., 2007; Pogrel, 2009; SriPathmanathan, 1990). Todavia, ainda são relatados casos desta complicação, seja decorrente de prática inadequada, movimento inesperado do paciente ou até mesmo defeitos de fabricação do instrumento em si. Esses incidentes são mais comuns em uso de agulhas com diâmetro menor e frequentemente ocorrem no centro (Nezafati \& Shahi, 2008; Thompson et al., 2003). Dentre os procedimentos de anestesia local realizada pelo cirurgião dentista, tem-se em destaque fraturas de agulha no procedimento de bloqueio do nervo alveolar inferior (Ferreira et al., 2016; Pogrel, 2009; Thompson et al., 2003).

Com isso, literatura traz inúmeros métodos para a resolução destes incidentes. Eles vão desde uso de hemostáticos finos na remoção imediata após a fratura, se ela estiver com a ponta visível. Até recursos como radiografias simples, radiográficas intraoperatórias com stents, a tomografia computadorizada e até uso de tecnologias mais avançadas, como scanners de TC helicoidal e iPlan Cranial 2.5 softwares (BrainLAB-Westchester, IL) (Ethunandan et al., 2007; Gerbino et al., 2013; Sri-Pathmanathan, 1990; Thompson et al., 2003).

No quesito de eficácia, agilidade de tempo cirúrgico, praticidade e segurança tanto para o paciente quanto para o operador, tem-se a opção da utilização do intensificador de imagens, o qual apresenta um método de detectar o corpo estranho em tempo real, sem expor o paciente à tomografia ou tomadas radiográficas, fornecendo uma localização dimensional com maior precisão, preservando estruturas anatômicas, reduzindo assim as possibilidades de complicações aos tecidos adjacentes. Visto que apenas os tecidos que se encontram em íntimo contato com o corpo estranho serão manipulados e que com tal precisão é possível a escolha de instrumentos mais específicos e seguros (de Queiroz et al.; Ferreira et al., 2016; MulinariSantos et al., 2018; Zhao et al., 2009),

Independente da técnica utilizada pelo operador para resolução do incidente, deve-se levar em consideração os sintomas do paciente e realizar a manobra visando a melhora do quadro. Além disso, é imprescindível que o paciente tenha 
conhecimento do que aconteceu e das medidas tomadas para resolução. Ele deve ter um acompanhamento adequado tanto dos sintomas, se presentes, quanto psicológico. Finalmente, a implicação médico-legal do incidente precisa ser levada em consideração, embora não deva ser primariamente acatada no gerenciamento (da Silva et al., 2020; Ethunandan et al., 2007; Glassman et al., 2009).

Este trabalho tem como objetivo relatar a utilização do intensificador de imagem para a remoção de agulha gengival fraturada na mucosa bucal durante o procedimento de anestesia local no nervo alveolar inferior em procedimento de exodontia do terceiro molar inferior esquerdo.

\section{Metodologia}

Trata-se de um estudo retrospectivo, descritivo e observacional no formato de nota técnica, representado por dois casos clínicos demonstrando a abordagem técnica através do intensificador de imagem para remoção de agulha fraturada e alojada em tecidos moles intrabucais. Os dados epidemiológicos, história da doença e registros fotográficos foram coletados através do prontuário físico e eletrônico, após autorização dos pacientes, mediante a assinatura do Termo de Consentimento Livre e Esclarecido (TCLE), seguindo os preceitos para publicação sem a identificação do paciente. Como meio de complemento e embasamento do estudo, foram feitas buscas na literatura utilizando o MeSH Database através da padronização de palavras-chave (Strange body; Needle; Traumatology). Com base na busca, foi feita uma breve discussão sobre as modalidades de diagnóstico e tratamento, visando os melhores resultados para os pacientes.

\section{Relato de Casos Clínicos}

O primeiro relato clínico abordado, trata-se de um paciente do sexo masculino, com 28 anos de idade, o qual foi encaminhado para o serviço de emergência do Hospital Santa Casa de Misericórdia de Araçatuba, após poucas horas de uma fratura de uma agulha gengival durante um procedimento odontológico. Em anamnese, o paciente não apresentava alterações sistêmicas, alergias ou uso diário de medicamentos, relatava queixas álgicas no local, e histórico do trauma a poucas horas da consulta hospitalar. Ao exame clínico extra oral, não apresentava alterações de normalidade, e ao exame intraoral apresentava aumento volumétrico sugestivo de edema em região de linha obliqua de mandíbula esquerda, com presença de material de sutura em região de trígono retromolar (Figura 1).

Figura 1 - (A) Acesso cirúrgico retromolar inferor esquerdo para localização e remoção da agulha fraturada; (B) Localização da agulha com a pinça hemostática com o auxílio do intensificador de imagem; (C) Agulha fraturada no intensificador de imagem imediatamente após remoção; (D) Agulha fraturada compara a lâmina de bisturi.

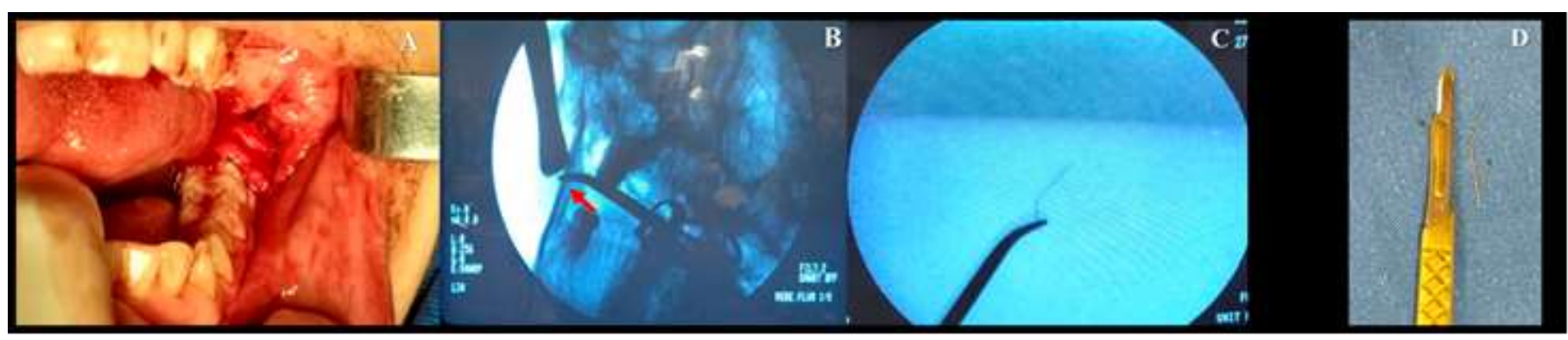

Fonte: Autores. 
O segundo relato clínico abordado, trata-se de uma paciente do sexo feminino, 48 anos de idade, sem comorbidades, alergias e uso contínuo de medicamentos. Apresentava histórico de fratura de agulha gengival na região de trígono retromolar à esquerda, há dois dias anteriores ao atendimento hospitalar, sem queixas álgicas. Ao exame clínico extra oral e intraoral, não apresentava alterações de normalidade. Ao exame tomográfico, apresentava imagem hipodensa, compatível com a agulha gengival, localizada paralelamente ao ramo mandibular, na região pterigomandibular, próximo ao forame do nervo alveolar inferior (Figura 2).

Figura 2 - (A) Tomografia Computadorizada (TC) em janela para tecidos ósseos e em corte sagital, demonstrando localização da agulha fraturada; (B) TC- corte axial demonstrando localização da agulha fraturada; (C) Localização da agulha no trans operatório com o auxílio do intensificador de imagem; (D) Acesso cirúrgico retromolar para a localização e remoção da agulha fraturada; (E) Agulha fraturada removida.

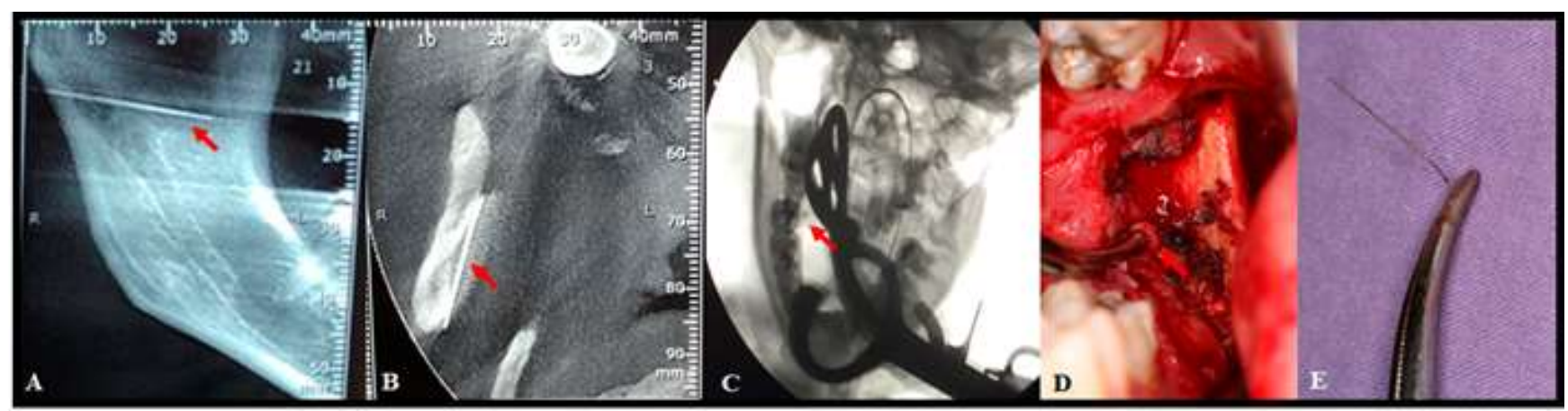

Fonte: Autores.

O tratamento de escolha para ambos os pacientes, foi um procedimento cirúrgico de urgência para remoção da agulha fraturada com auxílio do intensificador de imagem. Ambos os pacientes foram submetidos a anestesia geral, e a exploração cirúrgica para a localização da agulha, foi realizada por meio de uma incisão mucoperiosteal de distal do segundo molar até ramo mandibular do lado esquerdo.

Uma pinça clínica foi posicionada no interior dos tecidos como guia de localização sob o auxílio do intensificador de imagem (Simens AG, Modelo Multimobil 5c, Alemanha. As agulhas foram localizadas e removidas, em seguida foi realizado o tratamento da ferida cirúrgica com irrigação de solução de fisiológico $0,9 \%$ e suturado.

Ao acompanhamento pós operatório dos pacientes, apresentavam edema compatível com o trauma cirúrgico, sem sinais de infecção e sem queixas álgicas, no pós operatório imediato, assim como ao $3^{\circ}, 7^{\circ}$ e $15^{\circ}$ dia pós operatório, tendo alta da especialidade.

\section{Discussão}

A ocorrência de uma complicação como a fratura acidental de agulha gengival, com o alojamento em tecidos moles no atendimento odontológico, resulta em uma situação de estresse, tanto para o paciente quanto ao profissional (Faulkner \& Marshall, 1993; Gerbino et al., 2013; Marks et al., 1984; Ribeiro et al., 2014; Zeltser et al., 2002).

Diversos fatores podem ocasionar a fratura da agulha, desde defeitos na fabricação, realizar dobras na agulha ou em movimentos inesperados do paciente, como o fechamento repentino da boca. Após a imersão das agulhas descartáveis e ligas modernas, a ocorrência das fraturas das agulhas gengivais tornou-se mais raras, sendo mais voltadas ao domínio da técnica anestésica (Ethunandan et al., 2007; Ferreira et al., 2016; Pogrel, 2009). Os casos mais habituais envolvem as agulhas gengivais de menor diâmetro, que possuem maior deflexão e tendem a maior pressão aplicada sobre a seringa. Estes riscos 
podem ser exacerbados pela possibilidade de dobra da agulha gengival, a escolha do profissional e pela possível movimentação do paciente (Ethunandan et al., 2007).

A situação de urgência, caracterizada pelo alojamento da agulha em tecidos moles, requer uma rápida resolução no ponto de vista do paciente, porém, a decisão de realizar a remoção imediata ou tardia, varia de acordo com a experiência do profissional. A permanência do fragmento da agulha, pode desenvolver dor intensa, trismo e infecção, além da possibilidade do deslocamento do fragmento para outras estruturas, como o espaço lateral da faringe (Augello et al., 2011). Em ambos os casos abordados em nosso estudo, o profissional optou por encaminhar ao serviço especializado para realizar a remoção, e ambos os pacientes se apresentavam calmos e compreensivos diante da situação.

Neste contexto é possível discutir sobre os métodos convencionais, como o uso de radiografia e tomografia, que podem não obter sucesso e ainda trazer uma experiência mais traumática ao paciente. Em contrapartida, a fluorescência, que remete ao intensificador de imagem, possui uma alta taxa de sucesso ao fornecer uma localização precisa do corpo estranho e estruturas anatômicas adjacentes em tempo real, resultando em um procedimento minimamente invasivo, viável em questão de tempo cirúrgico, não traumático ao paciente, podendo levar em conta os aspectos estéticos ao minimizar as cicatrizes (Augello et al., 2011; Ferreira et al., 2016; Mulinari-Santos et al., 2018; Zhao et al., 2009). Este procedimento expõe o paciente a baixos níveis de radiação contínua, isto é, 0,5 a $2 \mathrm{~mA}$, sendo capaz de oferecer um retorno óptico de boa qualidade no transoperatório e não trazer danos ao paciente (Ethunandan et al., 2007; Faulkner \& Marshall, 1993; Ng et al., 2003; Rajkumar et al., 2014).

$\mathrm{O}$ intensificador de imagem descreve uma imagem gerada por uma fonte de raios $\mathrm{X}$ que é formada em uma tela fluorescente, convertendo a imagem dos raios x em uma imagem luminosa. Pode-se afirmar que a reprodução de imagem é fiel, visto que a intensidade de raios X aplicados são diretamente proporcionais à intensidade da luz (Canevaro, 2009; Faulkner \& Marshall, 1993). Outras vantagens sobre o intensificador de imagem partem da rápida aquisição e visualização da imagem, da facilidade de manejo da máquina fora do campo e da possibilidade de se obter imagens tiradas de forma rápida e em diferentes ângulos, sem alterar espacialmente o corpo estranho, facilitando sua localização (Nezafati \& Shahi, 2008; Thompson et al., 2003).

A escolha da anestesia geral facilita a execução do procedimento cirúrgico, proporcionando um campo de trabalho mais acessível e um resultado de qualidade, ocorrendo de forma rápida, efetiva e sem a possibilidade de movimentos bruscos ou reflexos do paciente (Canevaro, 2009; Glassman et al., 2009). Mulinari-Santos et al. (2018) relata uma tentativa de remoção de um anzol localizado em lábio inferior de maneira não palpável sob anestesia local e com auxílio de uma radiografia convencional pré operatória, porém houve dificuldade de localização do corpo estranho, o qual foi removido posteriormente sob anestesia geral e emprego de fluoroscopia, que proporcionou uma remoção rápida e atraumática do corpo estranho (Mulinari-Santos et al., 2018). Salientando que a escolha da técnica pré e transoperatória de localização e remoção do instrumento fraturado é importante para precisão no tratamento e para minimizar transtornos devido a intercorrência.

Por conta disso, o intensificador de imagem foi a escolha para tratamento de ambos os relatos mostrando ser a técnica mais eficiente e precisa comparada a radiografia e a tomografia computadorizada, mesmo que sejam eficientes e indicadas como guias de localização pré operatórios para diagnóstico e complementares a técnica de fluorescência (Augello et al., 2011). O que garantiu também que ambos os pós operatórios fossem bem sucedidos e sem intercorrências.

\section{Considerações Finais}

Mesmo ocorrendo de forma não habitual, os casos de fraturas de agulha gengival ainda acontecem em procedimentos odontológicos, e sua retirada é de grande importância, a partir da busca de métodos que garantam eficácia, agilidade de tempo cirúrgico, praticidade e segurança. Com isso, o intensificador de imagem revela-se como método de escolha, visto que, permite detectar um corpo estranho em tempo real, sem expor o paciente à tomografia ou tomadas radiográficas, fornecendo uma 
localização dimensional com maior precisão e sem lesar as estruturas anatômicas adjacentes. Para acautelar estes casos, é importante informar suas principais causas e conscientizar seus operadores da atenção à correta realização do procedimento de anestesia.

\section{Referências}

Augello, M., von Jackowski, J., Grätz, K. W., \& Jacobsen, C. (2011). Needle breakage during local anesthesia in the oral cavity--a retrospective of the last 50 years with guidelines for treatment and prevention. Clin Oral Investig, 15(1), 3-8. https://doi.org/10.1007/s00784-010-0442-6

Canevaro, L. (2009). Aspectos físicos e técnicos da radiologia intervencionista. Revista Brasileira de Física Médica, 3(1), 101-115.

da Silva, W. P. P., Mendes, B. C., de Jesus, K. G., Rios, B. R., Garbin, C. A. S., Junior, I. R. G., Souza, F. Á., \& Faverani, L. P. (2020). Facial trauma due to suicide attempt and its implications in a patient with psychological disorder: Brief Report. Research, Society and Development, 9(11), e44391110084e44391110084

de Queiroz, S. B. F., Moreira Jr, R., Farina, C. G., Moreira, R., da Silva, A. K. A., \& Coppedê, A. R. Uso Da Fluoroscopia Intraoperatória Para Guiar A Colocação De Implantes Zigomáticos.

Ethunandan, M., Tran, A. L., Anand, R., Bowden, J., Seal, M. T., \& Brennan, P. A. (2007). Needle breakage following inferior alveolar nerve block: implications and management. Br Dent J, 202(7), 395-397. https://doi.org/10.1038/bdj.2007.272

Faulkner, K., \& Marshall, N. W. (1993). The relationship of effective dose to personnel and monitor reading for simulated fluoroscopic irradiation conditions. Health Phys, 64(5), 502-508. https://doi.org/10.1097/00004032-199305000-00007

Ferreira, P., Ferreira, S., Fabris, A., Nogueira, L., Souza, F., \& Garcia Júnior, I. (2016). Remoção de agulha odontológica com intensificador de imagem. Journal of the Brazilian College of Oral and Maxillofacial Surgery, 2(1), 50-54. https://doi.org/10.14436/2358-2782.2.1.050-054.cre

Gerbino, G., Zavattero, E., Berrone, M., \& Berrone, S. (2013). Management of needle breakage using intraoperative navigation following inferior alveolar nerve block. Journal of Oral and Maxillofacial Surgery, 71(11), 1819-1824.

Glassman, P., Caputo, A., Dougherty, N., Lyons, R., Messieha, Z., Miller, C., Peltier, B., \& Romer, M. (2009). Special Care Dentistry Association consensus statement on sedation, anesthesia, and alternative techniques for people with special needs. Special Care in Dentistry, 29(1), 2-8. https://doi.org/10.1111/j.1754-4505.2008.00055.x

Marks, R. B., Carlton, D. M., \& McDonald, S. (1984). Management of a broken needle in the pterygomandibular space: report of case. J Am Dent Assoc, 109(2), 263-264. https://doi.org/10.14219/jada.archive.1984.0355

Mulinari-Santos, G., Bonardi, J. P., Fabris, A. L. d. S., Puttini, I. d. O., Coléte, J. Z., Duailibe-de-Deus, C. B., Faverani, L. P., Garcia Júnior, I. R., \& Souza, F. Á. (2018). Use of an image intensifier for the localization and removal of a foreign body in the lower lip. Archives of Health Investigation, 7(6). https://doi.org/10.21270/archi.v7i6.3008

Nezafati, S., \& Shahi, S. (2008). Removal of broken dental needle using mobile digital C-arm. J Oral Sci, 50(3), 351-353. https://doi.org/10.2334/josnusd.50.351

Ng, S. Y., Songra, A. K., \& Bradley, P. F. (2003). A new approach using intraoperative ultrasound imaging for the localization and removal of multiple foreign bodies in the neck. Int J Oral Maxillofac Surg, 32(4), 433-436. https://doi.org/10.1054/ijom.2002.0376

Pogrel, M. A. (2009). Broken local anesthetic needles: a case series of 16 patients, with recommendations. J Am Dent Assoc, 140(12), 1517-1522. https://doi.org/10.14219/jada.archive.2009.0103

Rajkumar, B., Boruah, L. C., Thind, A., Jain, G., \& Gupta, S. (2014). Dental Implant Placement using C-arm CT Real Time Imaging System: A Case Report. The Journal of Indian Prosthodontic Society, 14(1), 308-312.

Ribeiro, L., Ramalho, S., Gerós, S., Ferreira, E. C., e Almeida, A. F., \& Condé, A. (2014). Needle in the external auditory canal: an unusual complication of inferior alveolar nerve block. Oral surgery, oral medicine, oral pathology and oral radiology, 117(6), e436-e437.

Sri-Pathmanathan, R. (1990). The mobile X-ray image intensifier unit in maxillofacial surgery. British Journal of Oral and Maxillofacial Surgery, 28(3), 203206. https://doi.org/10.1016/0266-4356(90)90090-8

Thompson, M., Wright, S., Cheng, L. H., \& Starr, D. (2003). Locating broken dental needles. Int J Oral Maxillofac Surg, 32(6), 642-644. https://doi.org/10.1054/ijom.2003.0430

Zeltser, R., Cohen, C., \& Casap, N. (2002). The implications of a broken needle in the pterygomandibular space: clinical guidelines for prevention and retrieval. Pediatric dentistry, 24(2), 153-156.

Zhao, J., Chen, Y., Zeng, Q., He, X., Lu, W., Mei, Q., \& Li, Y. (2009). Removal of metallic foreign body in the soft tissue under fluoroscopy: 10 years of experiences. Nan fang yi ke da xue xue bao= Journal of Southern Medical University, 29(12), 2504-2505, 2509. 